\title{
Short Term Effects of Rhythmic Auditory Stimulation with Body Weight Supported Treadmill Training on Gait and Balance in Individuals with Incomplete Spinal Cord Injury
}

\author{
Kanika Singhal $^{1}$, Chitra Kataria ${ }^{2}$ \\ ${ }^{1}$ Research Student, MPT (Musculoskeletal), ISIC- Institute of Rehabilitation Sciences, \\ ${ }^{2}$ Research Guide, PhD in Physiotherapy and Rehabilitation, Chief of Rehabilitation Services, ISIC- Institute of \\ Rehabilitation Sciences, New Delhi \\ Corresponding Author: Chitra Katatria
}

\begin{abstract}
Background: Rhythmic auditory stimulation and body weight supported treadmill training both are standardized gait rehabilitation techniques. However, there is limited literature evaluating the effect of rhythmic auditory stimulation and its combination with gait training in spinal cord injury. Aim of this study is to determine the short term effectiveness of rhythmic auditory stimulation with body weight supported treadmill training on gait and balance in individuals with incomplete Spinal Cord Injury.
\end{abstract}

Method: A randomized control study design. 8 subjects with incomplete spinal cord injury who met the inclusion criteria were randomly allocated into two groups: Experimental and Control. Subjects in experimental group were given body weight supported treadmill training with rhythmic auditory stimulation. Subjects in Control Group were given Body weight supported treadmill training alone. Both the groups received conventional rehabilitation as well. Both groups received training for 30 minutes, five times a week for two weeks (10 sessions).

Outcome Measures: Gait parameters i.e. cadence, velocity, step length were measured using the Biodex Gait Trainer $2^{\mathrm{TM}}$, level of walking performance measured using Walking Index for Spinal Cord Injury II, and balance was evaluated using Prokin 252N ${ }^{\mathrm{TM}}$, Berg Balance Scale, and Activity specific Balance Confidence scale.
Results: No significant improvement was found on gait parameters i.e. cadence, velocity, step length which were measured using the Gait Trainer, level of walking performance measured using WISCI II, and balance which was evaluated using Prokin 252N ${ }^{\mathrm{TM}}$, Berg Balance Scale, and Activity specific Balance Confidence scale.

Conclusion: Rhythmic auditory stimulation didn't have any positive effect on gait training in incomplete spinal cord injured patients. Further studies are warranted to explore the entrainment effects of rhythmic auditory stimulation in spinal cord injured individuals on gait rehabilitation.

Keywords: Rhythmic Auditory Stimulation (RAS), Body Weight Supported Treadmill Training (BWSTT), Metronome, Incomplete spinal cord injury, Biodex Gait Trainer 2.0, Prokin 252N

\section{INTRODUCTION}

Spinal cord injury (SCI) is a high disabling injury (1). Spinal Cord Injury is a life-altering disability that profoundly impacts the physical, psychological, social, and financial aspects of a person's life (2). The aetiology of spinal cord injury may be traumatic or non-traumatic. The estimated global incidence of spinal cord injury is 40 to 80 new cases per million population per year, based on quality country-level incidence studies of spinal cord injury of all 
causes (3). In affected subjects the overall goal of rehabilitation interventions is to help them regain a level of independence compatible with a good quality of life. Although $80 \%$ of the patients can regain locomotor function, only a few of them can become functional walkers. They often walk slowly, with a long cycle time and small stride length. Ambulation entails the complex coordination of moving the legs, maintaining upright posture, maintaining balance, and responding to changes in the surroundings; injury to any part of this complex system can throw the delicate balance off (3).

Rhythmic auditory stimulation (RAS) is a standardized gait rehabilitation technique in which external rhythmic cueing facilitates training of intrinsically and biologically rhythmic movements, such as gait. The positive effects of rhythmic cueing have been observed in several neurological populations with gait deficits, including Parkinson's disease, stroke, cerebral palsy, traumatic brain injury and spinal cord injury (4). In patients with SCI, RAS could affect neuroplasticity (5). The body-weight supported treadmill training (BWSTT) enhance locomotor activity after a spinal cord injury. BWSTT techniques have been proposed as a promising way to help patients with SCI improve their gait (4).

Currently there is rare evidence showing effects of rhythmic auditory stimulation on gait and balance in patients with incomplete spinal cord injury. The previous studies have shown the immediate entrainment effects of rhythmic auditory stimulation on the gait parameters of cadence, velocity, and stride length in patients with incomplete spinal cord injury. The study by de l'Etoile et al. (2008), showed that the individuals with cervical injuries tended to increase cadence at both normal and fast walks, whereas individuals with thoracic injuries tended to increase both velocity and stride length during normal walk cueing than their own determination (6). The aim of this study is to evaluate the short term efficacy of RAS -
BWSTT on gait and balance in individuals with incomplete spinal cord injury improving their quality of life (4). And, the study by Amatachaya et al. (2009) showed that the use of external cues facilitated subjects to walk faster with a greater stride length, cadence and percent step symmetry (6).

\section{MATERIAL AND METHODS}

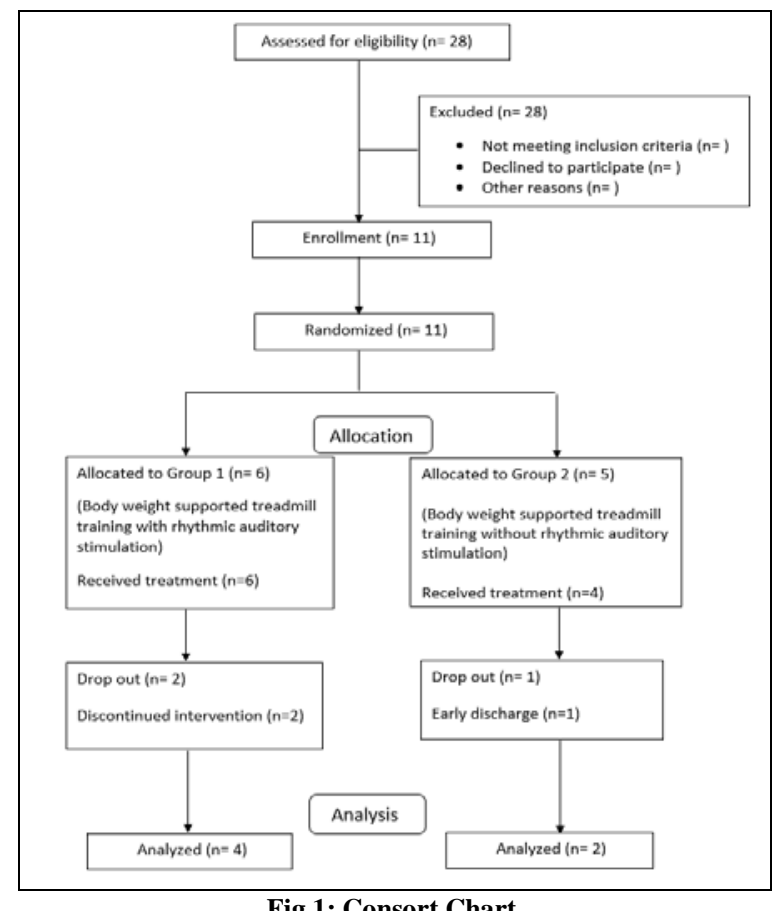

Fig 1: Consort Chart

The study was approved by the Institutional Research Review Committee and the Institutional Ethical Committee of the Indian Spinal Injuries Centre. A sample of 8 incomplete spinal cord injuries was taken who met the inclusion criteria: All subjects were given a detailed explanation of the procedure, and written consent was obtained (Fig: 1). Demographic data of subjects was collected (Table 1.1). Subjects were between 18 to 60 years of age. Motor incomplete traumatic and non-traumatic, non-progressive spinal cord injury classified as AIS-C and D and having achieved assisted standing a minimum of 1 week previously were included in the study. Exclusion criteria included orthopedic injuries that are unstable, osteoporosis with high risk of pathological fracture, cutaneous 

training on gait and balance in individuals with incomplete spinal cord injury.

lesions and/or pressure ulcers in areas where the harness or thigh straps are fitted, joint rigidity, asymmetry of lower-extremity length more than $2 \mathrm{~cm}$, pulmonary or heart disease requiring monitoring during exercise and body weight exceeding $150 \mathrm{~kg}$.

The subjects were randomly allocated into the following two groups. Group 1(Experimental group) ( $\mathrm{n}=4$ ) was given body weight supported treadmill training with rhythmic auditory stimulation (Fig 2) and group 2(Control group) $(n=4)$ was given Body weight supported treadmill training with rhythmic auditory stimulation (Fig 3). Rhythmic Auditory stimulation to experimental group was given by the instrument named metronome. Subjects were made to wear headphones for metronome beat auditory cues.

Table 1: Demographic details of subjects

\begin{tabular}{|l|l|l|}
\hline $\begin{array}{l}\text { Demographic } \\
\text { details }\end{array}$ & $\begin{array}{l}\text { Group 1 } \\
(\mathbf{n}=\mathbf{4}) \\
\text { Mean } \pm \text { S.D }\end{array}$ & $\begin{array}{l}\text { Group 2 } \\
\mathbf{( n = 4 )} \\
\text { Mean } \pm \text { S.D }\end{array}$ \\
\hline Age (Years) & $32.25 \pm 16.87$ & $32.00 \pm 4.08$ \\
\hline Weight (Kg) & $67.62 \pm 12.00$ & $66.50 \pm 13.67$ \\
\hline Height (cm) & $166.77 \pm 1.95$ & $172.50 \pm 3.31$ \\
\hline Gender & Male: 4 & Male: 4 \\
\hline ASIA (C/D) & ASIA C: 2 & ASIA C: 2 \\
& ASIA D: 2 & ASIA D: 2 \\
\hline Brace (KAFO/ None) & KAFO: 2 & KAFO: 3 \\
& None: 2 & None: 1 \\
\hline
\end{tabular}

Rhythm/tempo was set according to patient cadence measured on the treadmill. The intervention was carried out for 30 minutes, five times a week, for two weeks. The subjects were checked for any contraindications and ensured a safe, amicable environment to perform the intervention. Harness and the handrails during the treadmill training supported the patient from all sides to provide the patient confidence and sense of safety from any incidence of loss of balance.

The therapist made sure to be at the side while training and treadmill was stopped using STOP button whenever patient asked or needed a short break from the training.

\section{Data Analysis}

Mean \pm SD and count were used as descriptive measures for continuous and categorical variables respectively. Analysis was done for 8 individuals who completed the study. Mann-Whitney U test was done to check the homogeneity of the subjects in both the groups. Within group analysis between pre intervention and post intervention was performed using Wilcoxon Signed Rank Test. A significance level of $<$ 0.05 was fixed and the value of confidence interval was set at $95 \%$.

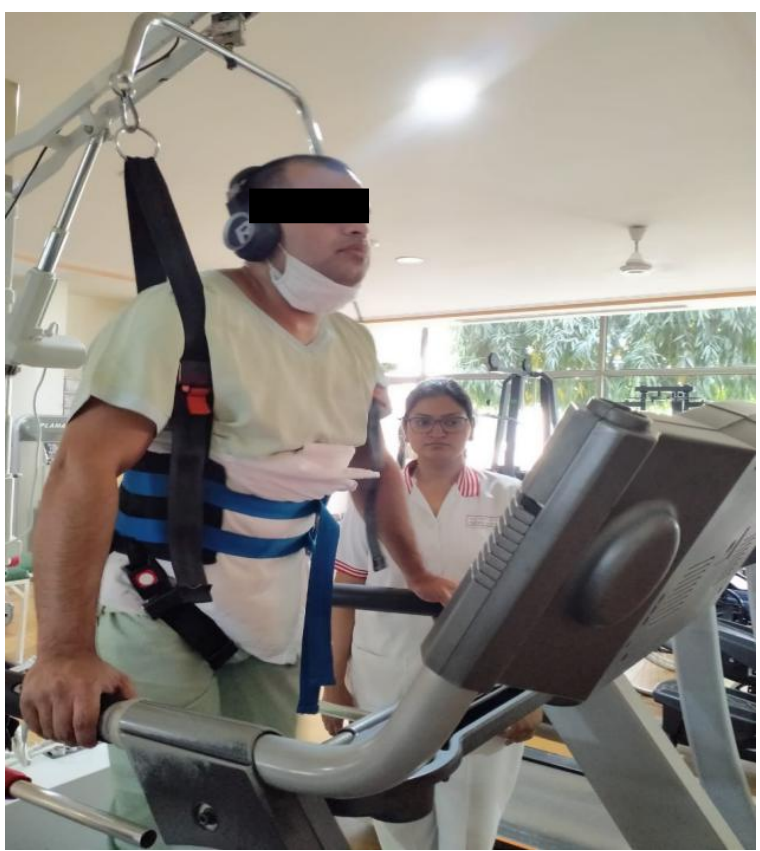

Fig 2: Body weight supported treadmill training with rhythmic auditory stimulation

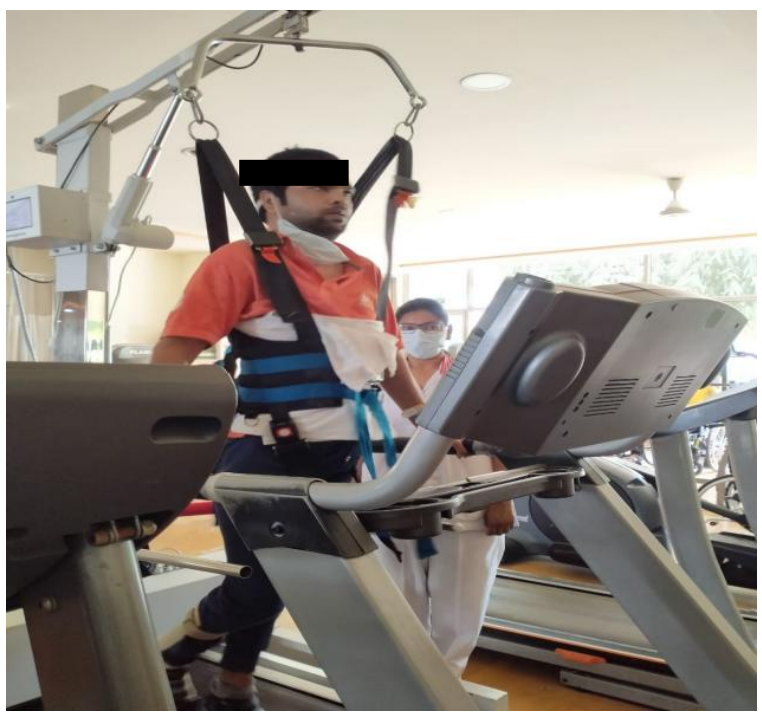

Fig 3: Control Group (Body weight Supported Treadmill Training)

\section{RESULTS}

A total of 8 subjects participated in the study. The 8 subjects were allocated 
randomly into two groups namely experimental group (Group 1) and control group (Group 2). The experimental group consists of 4 individuals (male $=4$, female $=0$ ) of which two were AIS C, two were AIS D. Two participants used braces. The participants in this group had a mean age (32.25 \pm 16.87) in years, height $(166.77 \pm 1.95)$ in $\mathrm{cm}$ and weight $(67.62 \pm$ $12.00)$ in $\mathrm{kg}$.

The control group consists of 4 individuals (male $=4$, female $=0$ ) of which two were AIS C, two were AIS D. Three participants used braces. The participants in this group had a mean age (32.00 \pm 4.08) in years, height $(172.50 \pm 3.31)$ in $\mathrm{cm}$ and weight $(66.50 \pm 13.67)$ in $\mathrm{kg}$. No significant differences were found between the age, height and weight for both the groups (Table 1). Hence the population in both the groups was homogenous.

Mann-Whitney U Test performed an analysis of the pre intervention scores of Group 1 and Group 2 at $p \leq 0.05$. There was a non significant difference between the pre intervention scores of both groups. Wilcoxon Sign Rank test for within group comparisons was done for difference of pre-test and post-test reading within the group for Cadence, Velocity, Step length (Left and Right), WISCI II, IEB, BBS and ABC Scale. No significant improvement was seen within both groups after two weeks.

\section{DISCUSSION}

Rhythmic Auditory Stimulation is considered a form of training that optimizes gait rehabilitation causing positive entrainment effect in neurological patients. Repetitive rhythmic cueing mediates motor control, and facilitates execution of expected motor responses. Body weight support treadmill training is another standardized technique for locomotor improvement in individuals with spinal cord injury.

\begin{tabular}{|l|l|l|l|l|l|l|}
\hline \multicolumn{7}{|c|}{ TABLE 2: Comparison of scores between experimental and control groups } \\
\hline Parameters & & GROUP 1 & & & GROUP 2 & \\
\hline & PRE & POST & p value & PRE & POST & p value \\
\hline Cadence & $49.25 \pm 10.56$ & $54.00 \pm 17.16$ & $0.285^{\mathrm{NS}}$ & $47.25 \pm 15.65$ & $52.25 \pm 17.32$ & $0.066^{\mathrm{NS}}$ \\
\hline Velocity & $0.550 \pm 0.41$ & $0.60 \pm 0.46$ & $0.317^{\mathrm{NS}}$ & $0.50 \pm 0.38$ & $0.50 \pm 0.38$ & $1.000^{\mathrm{NS}}$ \\
\hline Step length (rt) & $21.25 \pm 10.99$ & $26.25 \pm 13.02$ & $0.144^{\mathrm{NS}}$ & $17.50 \pm 9.29$ & $19.75 \pm 8.77$ & $0.465^{\mathrm{NS}}$ \\
\hline Step length (lt) & $19.50 \pm 12.15$ & $21.00 \pm 17.49$ & $0.593^{\mathrm{NS}}$ & $14.00 \pm 10.36$ & $15.00 \pm 10.98$ & $0.655^{\mathrm{NS}}$ \\
\hline WISCI II & $13.25 \pm 5.43$ & $13.75 \pm 5.50$ & $0.317^{\mathrm{NS}}$ & $10.50 \pm 5.44$ & $11.00 \pm 6.27$ & $0.317^{\mathrm{NS}}$ \\
\hline IEB & $24.75 \pm 13.22$ & $25.75 \pm 18.15$ & $0.713^{\mathrm{NS}}$ & $14.50 \pm 22.69$ & $16.50 \pm 21.01$ & $0.414^{\mathrm{NS}}$ \\
\hline BBS & $28.25 \pm 15.81$ & $29.50 \pm 16.60$ & $0.180^{\mathrm{NS}}$ & $22.25 \pm 13.91$ & $24.75 \pm 15.77$ & $0.102^{\mathrm{NS}}$ \\
\hline ABC SCALE & $58.28 \pm 22.06$ & $62.65 \pm 20.30$ & $0.357^{\mathrm{NS}}$ & $28.90 \pm 21.63$ & $36.71 \pm 26.26$ & $0.068^{\mathrm{NS}}$ \\
\hline
\end{tabular}

The aim of the present study was to determine the short term effectiveness of rhythmic auditory stimulation given using the device known as metronome when individual with incomplete spinal cord injury is performing body weight supported gait training on treadmill. The findings suggested that after 10 sessions over a period of 2 weeks, the subjects didn't show significant improvement on their gait parameters i.e. cadence, velocity, step length which were measured using the Gait Trainer, level of walking performance measured using WISCI II, and balance which was evaluated using Prokin $252 \mathrm{~N}^{\mathrm{TM}}$, BBS, and ABC scale.

\section{Effect of rhythmic auditory stimulation and gait training on Gait}

The findings are supported by a study conducted by De Eliote et al (2008), who also reported no interaction between cueing and tempo for any gait parameter (i.e. cadence, velocity and stride length) in patients with incomplete spinal cord injury when they examined the immediate entrainment effects of rhythmic auditory stimulation (4). Despite of statistical results in our study, entrainment ability was seen in patients in improving cadence during walking on treadmill. Subjects were able to increase their duration of training on each passing day. These results differ from the 
positive results of rhythmic auditory stimulation in gait training which have been seen in previous studies on other neurological populations such as multiple sclerosis, traumatic brain injury, stroke, cerebral palsy, Parkinson's disease (7-11).

Body weight support treadmill training (BWSTT) in particular is linked to improved muscle strength, kinematics, and spatiotemporal gait parameters, supporting central nervous system locomotor function recovery in iSCI patients. The role of BWSTT in the rehabilitation of walking independence in chronic iSCI patients is, however, unknown. Another study that examined the effects of a six-week body weight-support treadmill training (BWSTT) program on center-of-mass control and gait independence in chronic, incomplete spinal cord injury (iSCI) patients support our results on effect of training on WISCI II scores as their results reported that none of the patients obtained improved WISCI II scores pre- vs. post-intervention (12).

There is lack of positive effects of rhythmic auditory stimulation in our study on gait and balance in individuals with incomplete spinal cord injury when their gait training was combined with auditory stimulation for short term. However, this could be due to the reason that the sample size of the study was small and training period was for short term of 2 weeks due to limitations of covid situations. In our study, we enrolled all AIS C and D patients including those also who were not independent ambulatory. And provided them auditory stimulation during treadmill training with a harness for two weeks. Immediate or short term application of auditory stimulation might not improve the walking ability in spinal cord injured individuals. Also lack of a consistent effect may reflect the heterogeneous nature of spinal cord injuries, emphasizing the necessity of choosing suitable candidates who will benefit most from rhythmic cueing (4). Another possible reason for the ineffectiveness of rhythmic auditory stimulation could be that the music therapist was not included in the trial. Music can function as encouraging as well as distracting during the physical activity. Multiple auditory music beats should be there according to individual preference to increase his/her participation.

The auditory system, which works at rapid speed and provides a fine-grained depiction of the three-dimensional space around an individual, would be a natural source of balance-related information. Another key input for maintaining balance is proprioception (13). In our study we incorporated both the auditory and proprioceptive inputs in order to have their cumulative effect in improving balance. Previous research on the effects of rhythmic auditory stimulation on neurologic populations in gait training included participants who were already independent in ambulation. In our study, we also included individuals who needed assistive devices, required the assistance of another person during gait training, or could only walk short distances or for short periods of time. All of these differences in walking index for spinal cord injured people could have contributed to the study's inability to enhance balance in the short term with such a limited sample.

Both rhythmic auditory stimulation and locomotor training have been reported to have an effect on central pattern generators $(14,15)$. Higher centres (e.g. Basal ganglia) stimulate lower brainstem reticulospinal neurons, which activate spinal networks of nerve cells, i.e. CPGs, which generate the motor pattern. Sensory feedback to the CPG is an important aspect of the control system because it aids in the adaptation of the motor pattern to external stimuli (16). Combining the both techniques could have shown the desired result but the limited sample size might have contributed for inefficacy of treatment.

Another possible reason for the ineffectiveness of rhythmic auditory stimulation could be that the music therapist was not included in the trial. When contemplating music making as a kind of 
rehabilitative exercise for people with physical disabilities, two points of concern are: (a) their lack of knowledge with specialized musical devices, and (b) their incapacity to move in a controlled manner to manipulate the instruments. The music therapist and physical therapist can overcome these challenges by working together (17).

Music can function as encouraging as well as distracting during the physical activity. When determining which music to employ as an adjunct to therapy, the rate and tempo of the music must also be taken into account. Even if chosen music is effective, it may not be acceptable for all types of therapeutic activities. The music's beat must correspond to the rate at which the therapeutic activity is carried out, as well as the precise sections of the therapeutic activity. Music with a fast tempo, for example, may be distracting to a client in the early stages of gait training, such as while he or she is learning the stages of the gait cycle (heel strike, foot flat, heel off, toe off, and swing phases) (18). Multiple auditory music beats should be there according to individual preference to increase his/her participation. The ineffectiveness of using RAS with gait training could also be attributed to a lack of musical options and a variable tempo within two weeks.

Our study has some limitations. Study had small sample size. Also no follow up was done. The study was limited to one institute only. Further studies are warranted to explore the entrainment effects of rhythmic auditory stimulation in spinal cord injured individuals on gait rehabilitation.

\section{CONCLUSION}

Rhythmic auditory stimulation during body weight support treadmill training didn't show any significant improvement on their gait parameters i.e., cadence, velocity, step length which were measured using the Gait Trainer, level of walking performance measured using WISCI II, and balance which was evaluated using Prokin $252 \mathrm{~N}^{\mathrm{TM}}$, BBS, and $\mathrm{ABC}$ scale between and with both experimental and control groups over a period of two weeks. More studies on SCI patients are strongly needed to support RAS effects and to determine optimal intervention protocols that can be used in clinical practice in the future, because RAS is an efficient approach in these terms because it is viable, cheap, and can also be efficiently applied as a home-based intervention.

Acknowledgement: None

\section{Conflict of Interest: None}

\section{Source of Funding: None}

\section{Ethical Approval: Approved}

\section{REFERENCES}

1. Kang Y, Ding H, Zhou H, Wei Z, Liu L, Pan D, Feng S. Epidemiology of worldwide spinal cord injury: a literature review. Journal of Neurorestoratology. 2018;6(1):3.

2. Bhattarai M, Smedema SM, Maneewat K. An integrative review of factors associated with resilience post-spinal cord injury. Rehabilitation Counseling Bulletin. 2021 Jan;64(2):118-27.

3. Wall T, Feinn R, Chui K, Cheng MS. The effects of the Nintendo ${ }^{\mathrm{TM}}$ Wii Fit on gait, balance, and quality of life in individuals with incomplete spinal cord injury. The journal of spinal cord medicine. 2015 Nov 1;38(6):777-83.

4. Shannon K. The effect of rhythmic auditory stimulation on the gait parameters of patients with incomplete spinal cord injury: an exploratory pilot study. International Journal of Rehabilitation Research. 2008 Jun 1;31(2):155-7.

5. Mishra SS, Kimaya C, Kanchi V. Effects of Functional Proprioceptive Neuromuscular Facilitation with Mental Practice to Improve Activities of Daily Living in Syringomyelia Patient-A Case Report. Asian Journal of Case Reports in Medicine and Health. 2019 Jul 1:1-6.

6. Amatachaya S, Keawsutthi M, Amatachaya P, Manimmanakorn N. Effects of external cues on gait performance in independent ambulatory incomplete spinal cord injury 
Kanika Singhal et.al. Short term effects of rhythmic auditory stimulation with body weight supported treadmill training on gait and balance in individuals with incomplete spinal cord injury.

patients. Spinal Cord. 2009 Sep;47(9):66873.

7. Hurt CP, Rice RR, McIntosh GC, Thaut MH. Rhythmic auditory stimulation in gait training for patients with traumatic brain injury. Journal of music therapy. 1998 Dec 1;35(4):228-41.

8. Mainka S, Wissel J, Völler H, Evers S. The use of rhythmic auditory stimulation to optimize treadmill training for stroke patients: a randomized controlled trial. Frontiers in neurology. 2018 Sep 14;9:755.

9. Kwak EE. Effect of rhythmic auditory stimulation on gait performance in children with spastic cerebral palsy. Journal of music therapy. 2007 Oct 1;44(3):198-216.

10. Thaut MH, Rice RR, Braun Janzen T, HurtThaut CP, McIntosh GC. Rhythmic auditory stimulation for reduction of falls in Parkinson's disease: a randomized controlled study. Clinical rehabilitation. 2019 Jan;33(1):34-43.

11. Wittwer JE, Webster KE, Hill K. Rhythmic auditory cueing to improve walking in patients with neurological conditions other than Parkinson's disease-what is the evidence?. Disability and rehabilitation. 2013 Jan 1;35(2):164-76.

12. Covarrubias-Escudero F, Rivera-Lillo G, Torres-Castro R, Varas-Díaz G. Effects of body weight-support treadmill training on postural sway and gait independence in patients with chronic spinal cord injury. The journal of spinal cord medicine. 2019 Jan 2;42(1):57-64.

13. Stevens MN, Barbour DL, Gronski MP, Hullar TE. Auditory contributions to maintaining balance. Journal of Vestibular Research. 2016 Jan 1;26(5-6):433-8.

14. O'Sullivan SB, Schmitz TJ, Fulk G. Physical rehabilitation. FA Davis; 2019 Jan 25.

15. Thaut M, Hoemberg V, editors. Handbook of neurologic music therapy. Oxford University Press (UK); 2014.

16. Grillner S. Neurobiological bases of rhythmic motor acts in vertebrates. Science. 1985 Apr 12;228(4696):143-9.

17. Paul S, Ramsey D. Music therapy in physical medicine and rehabilitation. Australian Occupational Therapy Journal. 2000 Sep;47(3):111-8.

18. Sandness MI. The role of music therapy in physical rehabilitation programs. Music Therapy Perspectives. 1995 Nov 1;13(2):7681.

How to cite this article: Singhal K, Kataria C. Short term effects of rhythmic auditory stimulation with body weight supported treadmill training on gait and balance in individuals with incomplete spinal cord injury. International Journal of Science \& Healthcare Research. 2021; 6(4): 191-197. DOI: https:// doi.org/10.52403/ijshr.20211027 